ARTICLE

Received 3 Aug 2015 | Accepted 28 Mar 2016 | Published 29 Apr 2016

DOI: $10.1038 /$ ncomms 11443

OPEN

\title{
Quantum Einstein-de Haas effect
}

\author{
Marc Ganzhorn ${ }^{1, \dagger}$, Svetlana Klyatskaya ${ }^{2}$, Mario Ruben ${ }^{2,3} \&$ Wolfgang Wernsdorfer ${ }^{1}$
}

The classical Einstein-de Haas experiment demonstrates that a change of magnetization in a macroscopic magnetic object results in a mechanical rotation of this magnet. This experiment can therefore be considered as a macroscopic manifestation of the conservation of total angular momentum and energy of electronic spins. Since the conservation of angular momentum is a consequence of a system's rotational invariance, it is valid for an ensemble of spins in a macroscopic ferromaget as well as for single spins. Here we propose an experimental realization of an Einstein-de Haas experiment at the single-spin level based on a single-molecule magnet coupled to a nanomechanical resonator. We demonstrate that the spin associated with the single-molecule magnet is then subject to conservation of total angular momentum and energy, which results in a total suppression of the molecule's quantum tunnelling of magnetization.

\footnotetext{
${ }^{1}$ Institut Néel, CNRS \& Université Joseph Fourier, BP 166, 25 Avenue des Martyrs, 38042 Grenoble Cedex 9, France. ${ }^{2}$ Institut of Nanotechnology (INT), Karlsruhe Institute of Technology (KIT), 76344 Eggenstein-Leopoldshafen, Germany. ${ }^{3}$ Institut de Physique et Chimie des Matériaux de Strasbourg (IPCMS), CNRS-Université de Strasbourg, 67034 Strasbourg, France. †Present address: Department of Physics, University of Basel, Klingerlbergstrasse 82, 4056 Basel, Switzerland. Correspondence and requests for materials should be addressed to W.W. (email: wolfgang.wernsdorfer@neel.cnrs.fr).
} 
$\mathrm{O}$ ne hundred years ago it has been discovered that a change of magnetization in a macroscopic magnetic object results in a mechanical rotation of this magnet. The effect, known as Einstein-de Haas or Richardson effect, demonstrates that a spin angular momentum in the magnet compensates for the mechanical angular momentum associated with its rotation ${ }^{1-3}$. The experiment is therefore a macroscopic manifestation of the conservation of total angular momentum and energy in eletronic spins. According to Noether's theorem ${ }^{4}$, conservation of angular momentum follows from a system's rotational invariance and would be valid for the ensemble of spins in a macroscopic ferromagnet as well as for an individual spin. It has been recently proposed that single-spin systems would therefore manifest an Einstein-de Haas effect at the quantum level ${ }^{5-7}$.

Here we describe an experimental realization of a quantum Einstein-de Haas experiment, which consists of a single-molecule magnet (SMM) attached to a carbon nanotube (CNT) mechanical resonator. We demonstrate that the spin associated with the single-molecule magnet is then subject to conservation of total angular momentum and energy. At small applied fields, this results in a total suppression of the molecule's quantum tunnelling of magnetization, whereas at higher magnetic field the magnetization reversal occurs via a direct transition between the electronic spin states for a transition energy matching the phonon energy of the CNT mechanical resonator. These findings demonstrate the importance of angular momentum conservation in magnetic nanostructures and are crucial to the field of molecular quantum spintronics. ${ }^{8}$ Indeed, the presented suppression of quantum tunnelling could help to increase the spin lifetime $\mathrm{T}_{1}$ of SMMs and other nanomagnets.

\section{Results}

Single-molecule magnets. A SMM is a nanomagnet, consisting of exchange-coupled magnetic centres, typically a rare earth or transition metal ion, embedded in a shell of organic ligands 8,9 . The ligands are designed to promote the molecule's coupling to the environment while protecting and enhancing the ion's magnetic properties. Rare earth-based SMMs can have a large magnetic ground state with a spin $S>1$ and a strong magnetic anisotropy defining the molecule's easy axis of magnetization ${ }^{10,11}$. The spin physics can thus be described by a 'giant spin' Hamiltonian of the form

$$
\mathcal{H}=D S_{z}^{2}+E\left(S_{x}^{2}-S_{y}^{2}\right)+g \mu_{\mathrm{B}} \mu_{0} \mathbf{S} \cdot \mathbf{H}
$$

where $S_{x}, S_{y}, S_{z}$ are the spin components, $D$ the axial and $E$ the transverse magnetic anisotropy constants (with $D \gg E$ ), and $g \mu_{\mathrm{B}} \mu_{0} \mathbf{S} \cdot \mathbf{H}$ the Zeeman energy associated with an applied external magnetic field. At low temperatures $\left(k_{\mathrm{B}} T \ll D S_{z}^{2}\right)$, the SMM can be described as a two-level system ${ }^{10,11}$ and the magnetization reversal of the SMM can then occur via two different processes ${ }^{10,11}$. During a direct transition process (DT), the SMM's magnetization reversal is enabled by the excitation of phonons with a finite energy in the molecule's environment at higher magnetic fields ${ }^{10,12}$. Alternatively, the magnetization reversal occurs by quantum tunnelling of magnetization (QTM) between up and down spin polarization at low magnetic fields ${ }^{10,13}$. The tunnelling probability of the molecule is given by the magnitude of the tunnel splitting $\Delta$ that arises from the transverse magnetic anisotropy component ${ }^{10,13}$.

According to Noether's theorem, both reversal processes have to conserve the total angular momentum as well as energy. For instance, a free SMM that is completely isolated from its environment cannot exchange angular momentum and energy with the latter. Magnetization reversal would be fully supressed in order to conserve total angular momentum and energy ${ }^{14}$. On the other hand, it has been predicted that magnetization reversal can occur in a SMM coupled to a nanomechanical resonator ${ }^{5-7}$. In analogy to a classical Einstein-de Haas effect (Fig. 1a), the magnetization reversal results in a rotation of the SMM to satisfy conservation of angular momentum and energy, and generates a quantized phonon mode in the resonator (Fig. 1b). However, vibrational or rotational modes in nanomechanical resonators carry a non-zero angular momentum and their energy $\left(\hbar \omega_{\mathrm{r}}\right.$ : $\mathrm{MHz}-\mathrm{GHz}$ (ref. 15)) is typically larger then the tunnel splitting $(\Delta: \mathrm{kHz}$ (ref. 13)). Consequently, QTM in a SMM coupled to a nanoresonator would be suppressed to conserve both energy and angular momentum ${ }^{16,17}$. Hence, magnetization reversal in the SMM only occurs by a direct transition and the excitation of a resonator mode. In this letter, we report on experimental evidence for such a quantum Einstein-de Haas effect and demonstrate that conservation of total angular momentum and energy fully suppresses QTM in a SMM coupled to a carbon nanotube nanoresonator.

$\mathrm{TbPc}_{2}$ single-molecule magnet. The pyrene-subtituted bis(phthalocyaninato)terbium(III) molecule $\left(\mathrm{TbPc}_{2}\right)$ is a rare earth SMM in which the magnetic moment is carried by a single $\mathrm{Tb}^{3+}$ ion sandwiched between two organic phthalocyanine $(\mathrm{Pc})$ ligand planes $^{11,12}$ (see Methods for synthesis). The $\mathrm{TbPc}_{2}$ has a $S=1 / 2$ radical delocalized over the Pc ligand planes. Due to $\pi-\pi$ interaction, this radical can easily hybridize with the $\pi$-electrons of any form of $s p_{2}$-carbon without affecting the magnetic properties of the $\mathrm{Tb}^{3+}$ ion ${ }^{11,12,18,19}$. The highly anisotropic $4 f$ shell of the $\mathrm{Tb}^{3+}$ ion and its intrinsically strong spin-orbit coupling result in a magnetic ground state of $J=6$ and a pronounced uniaxial magnetic anisotropy (Fig. 2a). The ground-
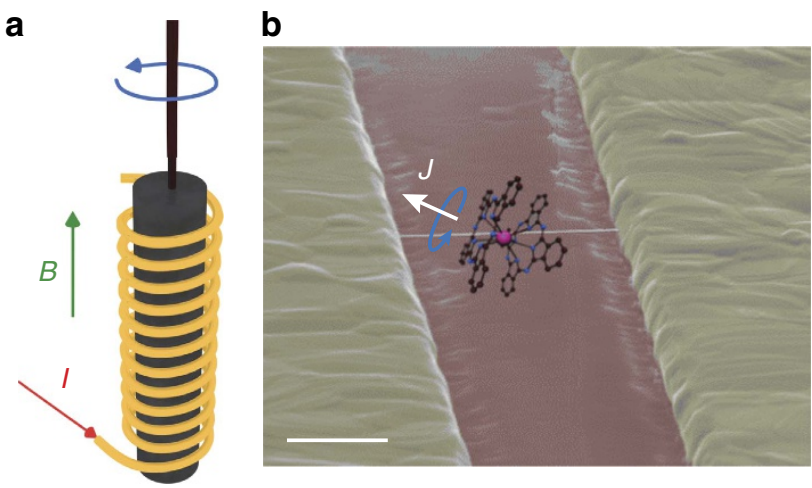

Figure 1 | Set-up of the Einstein-de Haas experiments. (a) For the classical Einstein-de Haas experiment, a magnetic material is suspended with the aid of a thin string inside a magnetic field coil. When the magnetic field $B$ is increased by the application of an electric current / through the field coil, the magnetic material is magnetized. In order to keep the total magnetic moment of the magnetic material constant, the latter rotates. This classical Einsteinde Haas effect demonstrates that the spin angular momentum is of the same nature as the angular momentum of rotating bodies as conceived in classical mechanics. (b) For the quantum Einstein-de Haas experiment, the false colour scanning electron micrograph shows a suspended carbon nanotube with a local metallic backgate (red) functionalized with a pyrene-substituted bis(phthalocyaninato)terbium(III) (hereafter $\mathrm{TbPc}_{2}$ ) single-molecule magnet (shown as a chemical structure overlaid on the image, not to scale, pyrene omitted for clarity). Due to conservation of the total angular momentum, the magnetization reversal of $J=6$ (white arrow) in a magnetic field results in a rotation of the single-molecule magnet (blue arrow), thus generating a quantized phonon mode in the carbon nanotube nanoelectromechanical resonator. Scale bar, $500 \mathrm{~nm}$. 


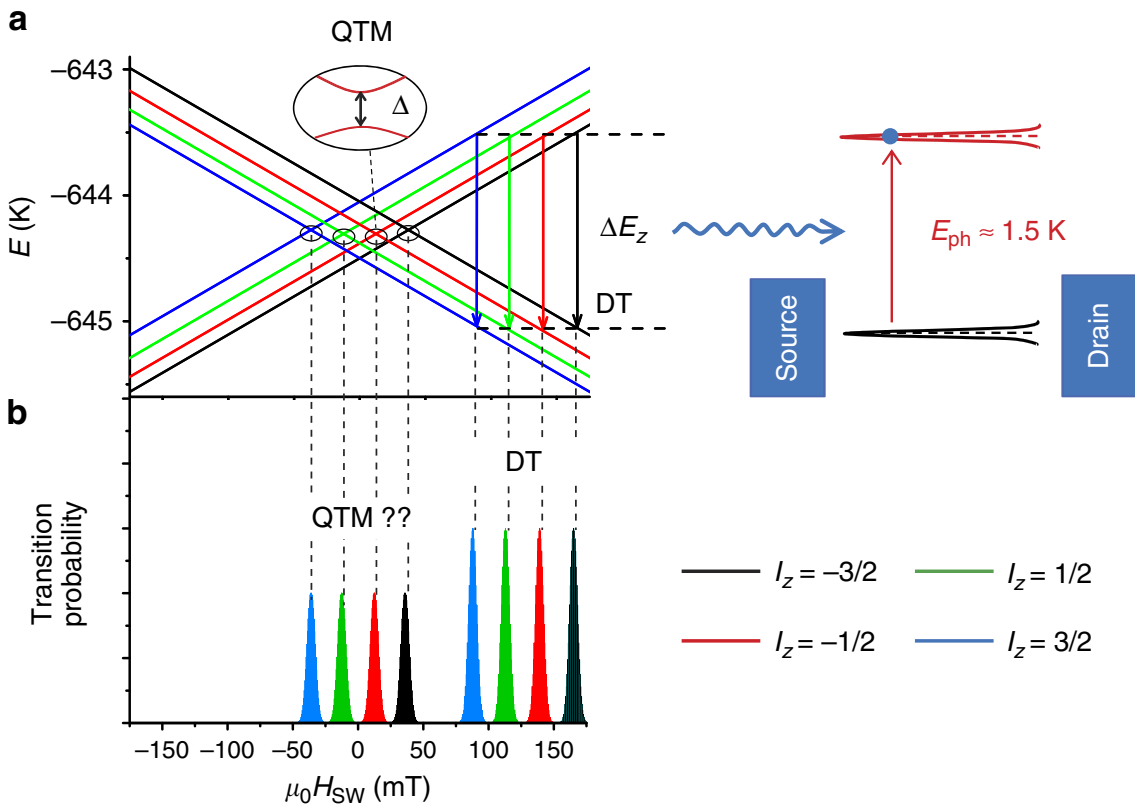

Figure 2 | Energy schematics of the quantum Einstein-de Haas experiment. (a) Zeeman diagram of the ground-state energy levels of the TbPc $\mathrm{C}_{2}$ molecule with the magnetic field applied parallel to the easy axis of magnetization. Because of a strong hyperfine interaction with the Tb nuclear spin, the electron's up and down states are each split into four energy levels, labelled with the nuclear spin states $I_{z}= \pm 1 / 2, \pm 3 / 2$. At higher magnetic field, the magnetization reversal can occur via a direct transition (DT) between the electronic spin states for a transition energy $\Delta E_{z}$ matching the phonon energy $E_{\mathrm{ph}}=1.5 \mathrm{~K}$. Note that, for all transitions, the nuclear spin is preserved and therefore their positions reveal the nuclear spin states. At small fields, off-diagonal terms in the spin Hamiltonian lead to avoided energy level crossings (shown in the inset), enabling in principle tunnelling of the electronic spin. However, QTM is suppressed due to the absence of a phonon mode in the carbon nanotube resonator, which can absorb the angular moment and has in addition a very low energy, being of the order of magnitude of the tunnel splitting $\Delta \sim 1 \mu \mathrm{K}$. (b) Magnetization reversal histograms of the $\mathrm{TbPc}_{2}$ molecule coupled to the phonon mode of the carbon nanotube resonator. According to (a) one would expect the absence of QTM events (labelled by 'QTM ??') due to the coupling to the nanoresonator.

state doublet $J_{\mathrm{z}}= \pm 6$ is separated from the excited states by several hundreds of Kelvin, which makes the $\mathrm{TbPc}_{2}$ an Ising-like spin system at low temperature $(T<5 \mathrm{~K})$ and small magnetic field $(B<10 \mathrm{~T})^{11,12}$. A strong hyperfine interaction with the nuclear spin $I=3 / 2$ of the $\mathrm{Tb}^{3+}$ ion splits the ground state doublet $J_{\mathrm{z}}= \pm 6$ into four states each (Fig. 2a). Finally, the ligand field generates a small transverse magnetic anisotropy resulting in avoided level crossings (black circles in Fig. 2a) ${ }^{11,12}$.

Magnetization reversal of a SMM. According to the LandauZener-Stueckelberg-Majorana formalism ${ }^{20-23}$, the magnetization reversal will occur via QTM at one of the four avoided level crossings around zero field (black circles in Fig. 2a), with a probability $P_{\mathrm{QTM}}$ given by

$$
P_{\mathrm{QTM}}=1-\exp \left(-\alpha \frac{\Delta^{2}}{v}\right)
$$

with the tunnel splitting $\Delta, v=\mathrm{d} H / \mathrm{d} t$ the magnetic field sweep rate and $\alpha$ a coefficient specific to the SMM. Alternatively, the spin reversal occurs via a direct transition with a probability $1-P_{\mathrm{QTM}}$ at a larger value of magnetic field, the so-called switching field $\mu_{0} H_{\text {Sw }}$. As demonstrated in ref. 12, the switching field $\mu_{0} H_{\mathrm{SW}}$ is determined by the energy of the phonon(s) involved in the process.

Owing to conservation of total angular momentum, the magnetization reversal from $J_{z}=6$ (white arrow in Fig. 1b) to $J_{z}=-6$ of a $\mathrm{TbPc}_{2} \mathrm{SMM}$ grafted to a suspended carbon nanotube (see Methods for sample fabrication) results in a rotation of the SMM around its magnetic easy axis (blue arrow Fig. 1b), thus generating a quantized longitudinal phonon mode in the carbon nanotube resonator. We demonstrated in ref. 12 that the magnetization reversal in such a supramolecular spintronic device can occur via direct transition from the nuclear spin states of the $\mathrm{Tb}^{3+}$, for a transition energy $\Delta E_{z}$ matching the phonon energy $E_{\mathrm{ph}}=1.5 \mathrm{~K}$ (Fig. 2a,b). As we will demonstrate in the following, the probability for QTM on the other hand is fully suppressed in the absence of a phonon mode, which can absorb the angular moment and has in addition a very low energy, being of the order of magnitude of the tunnel splitting $\Delta \sim 1 \mu \mathrm{K}$ (Fig. 2a,b).

Quantum Einstein-de Haas experiment. Using an electronic readout (see Methods), we study the magnetization reversal of the $\mathrm{TbPc}_{2} \mathrm{SMM}$ coupled to the carbon nanotube resonator as a function of the magnetic field sweep rate $v$, the transverse magnetic field component $\mu_{0} H_{\perp}$ and the temperature $T$. Figure $3 \mathrm{a}$ shows histograms of the switching fields $\mu_{0} H_{\text {Sw }}$ extracted from 200 back and forth magnetic field sweeps, for four different magnetic field sweep rates $v$. According to equation (1), one can alter the QTM probability by tuning the sweep rate of the magnetic field. However, for sweep rates $v$ ranging from 10 to $150 \mathrm{mT} \mathrm{s}^{-1}$, we observe a $100 \%$ probability for a magnetization reversal via direct transitions, whereas a zero probability for a reversal via QTM is found.

Furthermore, it was shown in $\mathrm{Mn}_{4}$ SMM crystals ${ }^{24}$ and individual $\mathrm{TbPc}_{2} \mathrm{SMM}$ integrated in carbon nanotube transistors $^{13}$ that a transverse magnetic field modifies the tunnel splitting $\Delta$ and thus the magnet's QTM probability $P_{\mathrm{QTM}}$. Specifically, a transverse magnetic field of a few $100 \mathrm{mT}$ can modify the QTM probability $P_{\mathrm{QTM}}$ of an individual $\mathrm{TbPc}_{2}$ by 

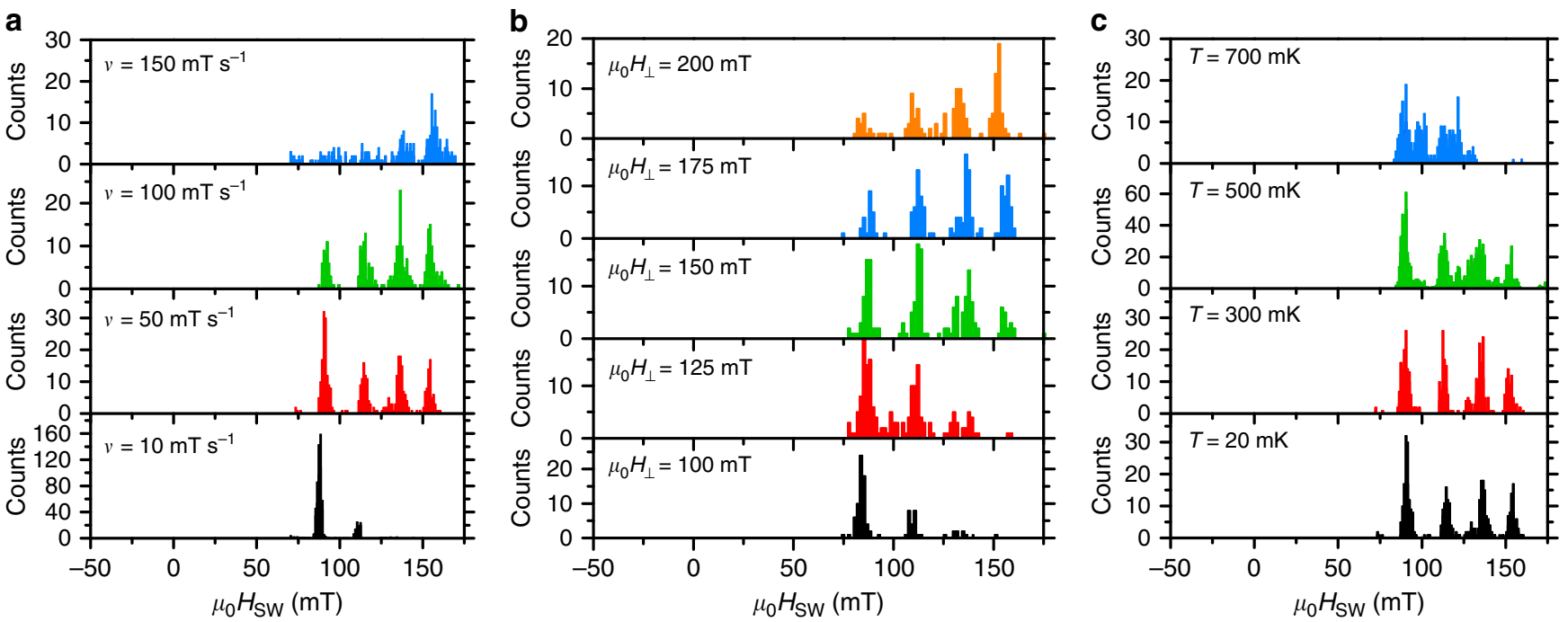

Figure 3 | Magnetization reversal histograms of the quantum Einstein-de Haas experiment. Histograms of the swichting fields $\mu_{0} H_{\mathrm{SW}}$ for several (a) field sweep rates, (b) transverse magnetic fields and (c) temperatures. Each histogram corresponds to 200 field sweeps from negative to positive magnetic fields. No QTM is observed around zero magnetic field, resulting in a $100 \%$ probability for a magnetization reversal via a direct transition of the electronic spin state of the $\mathrm{Tb}^{3+}$ ion, which depends on the four nuclear spin states. Note that if the SMM had tunnelled at small fields without an observable conductance jump, it could not make a direct transition because it would already be in the ground state.

a factor of 2-3 (ref. 13). Figure $3 \mathrm{~b}$ shows the switching field distribution of the $\mathrm{TbPc}_{2}$ SMM coupled to the carbon nanotube resonator for transverse magnetic fields $\mu_{0} H_{\perp}$ ranging from 100 to $200 \mathrm{mT}$. We find a probability for QTM equal to zero with a $100 \%$ probability of magnetization reversal of the SMM via direct transitions (Fig. 3b).

Finally, we consider the magnetization dynamics of the $\mathrm{TbPc}_{2}$ SMM coupled to the carbon nanotube resonator as a function of temperature. As a matter of fact, additional phonon modes can be excited in the carbon nanotube resonator at higher temperatures and it has been demonstrated that tunnelling processes in a SMM can also be thermally activated ${ }^{25}$. However, the total suppression of QTM persists for temperatures up to $T=700 \mathrm{mK}$, whereas direct transitions of the electronic spin of the $\mathrm{TbPc}_{2} \mathrm{SMM}$ can still occur with a dependance on the nuclear spin state (Fig. $3 \mathrm{c}$ ).

These findings can thus be considered a direct manifestation of the conservation of angular momentum and energy, that is, the quantum Einstein-de Haas effect, which fully suppresses QTM of the $\mathrm{TbPc}_{2}$ in the absence of a phonon mode in the carbon nanotube resonator, capable of absorbing the change of angular momentum $\Delta J_{\mathrm{z}}=12$ and the energy $\Delta \sim 1 \mu \mathrm{K}$ associated with the QTM process in $\mathrm{TbPc}_{2}$ SMM. Indeed, we only observe a quantized longitudinal stretching mode phonon in the carbon nanotube resonator, with an energy $E_{\text {long }}=1.5 \mathrm{~K}$ much larger than the tunnel splitting $\Delta \sim 1 \mu \mathrm{K}$. In general, the expected energy for a bending-mode phonon ( $E_{\text {bend }} \sim 0.01 \mathrm{~K}$, ref. 26 ), longitudinal stretching-mode phonon $\left(E_{\text {long }} \sim 1 \mathrm{~K}\right)$ or a radial breathing-mode phonon $\left(E_{\mathrm{RBM}} \sim 500 \mathrm{~K}\right.$, ref. 26$)$ in carbon nanotube resonators is orders of magnitude larger than the tunnel splitting $\Delta$. Consequently, QTM should be always suppressed in $\mathrm{TbPc}_{2} \mathrm{SMM}$ coupled to a carbon nanotube resonator. Figure 4 shows the magnetization reversal of four different $\mathrm{TbPc}_{2}$ molecules coupled to different carbon nanotube resonators. Indeed we observe the full suppression of QTM on all four molecules. The direct transition of each molecule occurs with a probability of $100 \%$, that is, it cannot have made a tunnel transition at small fields. However, the transitions of the four samples occur at different switching fields $\mu_{0} H_{\mathrm{SW}}$, which is related to different energies of the involved phonon mode. It

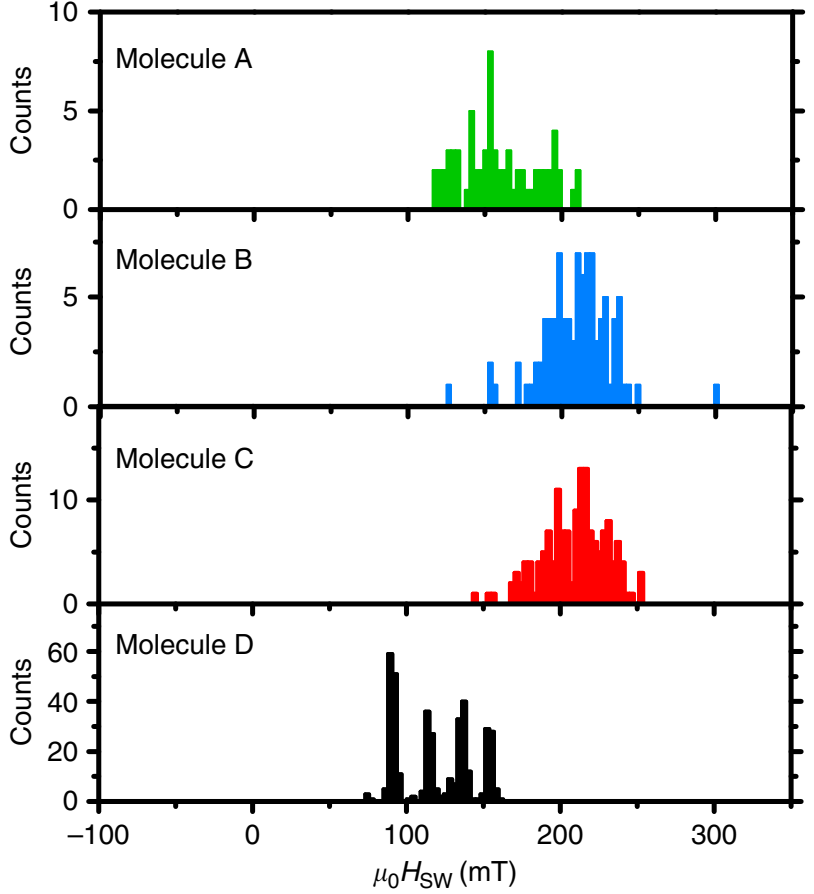

Figure 4 | Magnetization reversal histograms of the quantum Einstein-de Haas experiment on different $\mathbf{T b P c}_{\mathbf{2}}$ molecules. The histograms are recorded at $T=20 \mathrm{mK}$ and a magnetic field sweep rate of $50 \mathrm{mTs}^{-1}$. It should be noted that the nuclear spin states cannot be resolved in molecules $\mathrm{A}-\mathrm{C}$, which can be attributed to the presence of strong fluctuating magnetic fields resulting in linewidth broadening, to weak interaction of the nuclear spin with its environment, or to a small quality factor of the CNT resonator. No QTM is observed around zero magnetic field, but a $100 \%$ probability for a magnetization reversal via a direct transition of the electronic spin state of the $\mathrm{Tb}^{3+}$ ion.

should be pointed out that we do not observe stochastic fluctuations between $J_{z}=6$ and $J_{z}=-6$, indicating that we are not resonantly exciting the phonon mode. 


\section{Discussion}

The robustness of the observed phenomena towards external manipulation further corroborates theoretical predictions of a quantum Einstein-de Haas effect, that is, that single-spin dynamics such as quantum spin tunnelling are ultimately governed by conservation of total angular momentum. Note in particular that all kinds of structural defects of the device or molecule would enhance the tunnel rates because any symmetry breaking induces transverse anisotropy terms in the spin Hamiltonian, which increases the mixing of the spin wave functions. This results in an increase of the tunnel splitting and thus of the tunnel rates.

In conclusion, we can confirm theoretical predictions of a quantum Einstein-de Haas effect, which states that total angular momentum and energy have to be conserved for individual spins $s^{5-7}$. The effect manifests itself as a total suppression of QTM in a SMM coupled to a carbon nanotube nanoresonator and is robust against changes in temperature, magnetic field or phonon energy of the nanoresonator. It was recently predicted that such a quantum Einstein-de Haas effect effectively screens the molecular spin against quantum fluctuations, therefore enabling the coherent spin manipulation on a single-phonon level ${ }^{16}$.

\begin{abstract}
Methods
Nanofabrication. The suspended carbon nanotube resonators are built in an ultraclean, bottom-up fabrication process. For this purpose, a 1- $\mu \mathrm{m}$-wide metallic local gate is patterned by optical deep ultraviolet lithography and subsequent e-beam evaporation of Mo $(20 \mathrm{~nm})$ on a degenerately p-doped silicon wafer with a 300 -nm-thick layer of thermal $\mathrm{SiO}_{2}$. A layer of $100 \mathrm{~nm}$ of $\mathrm{Al}_{2} \mathrm{O}_{3}$ is then deposited by atomic layer deposition. Using optical deep ultraviolet lithography and e-beam evaporation of Mo $(20 \mathrm{~nm})$ and Pt $(160 \mathrm{~nm})$, source-drain electrodes are aligned above the local gate. Suspended carbon nanotubes are finally grown by chemical vapor deposition (CVD) at $800{ }^{\circ} \mathrm{C}$ from a $\mathrm{CH}_{4}$ feedstock and $\mathrm{Fe} / \mathrm{Mo}$ catalyst spots patterned on the source-drain electrodes next to the junction. The lengths of the carbon nanotubes are estimated to a range of $800-900 \mathrm{~nm}$ (ref. 12). In a second step, pyrene-substituted $\mathrm{TbPc}_{2}$ SMMs are synthesized by a divergent multistep protocol utilizing two different phthalocyaninato lithium salts and $\left[\mathrm{Tb}(\mathrm{acac}) 3^{\star} 2 \mathrm{H}_{2} \mathrm{O}\right](\mathrm{acac}=$ acetylacetonato $)$ in 1:1:1 ratio ${ }^{18}$. Finally, the $\mathrm{TbPc}_{2}$ powder is dissolved in a solution of dichloromethane and drop-casted onto the sample. The droplet is subsequently dried in a critical point dryer, to avoid destruction of the suspended carbon nanotubes through capillarity effects ${ }^{12}$.
\end{abstract}

Detection of SMM magnetization reversal. Magnetic field sweeps are performed along the $\mathrm{TbPc}_{2}$ easy axis from negative to positive magnetic field values and back, while monitoring the differential conductance in the carbon nanotube resonator. The magnetization reversal of the $\mathrm{Tb}^{3+}$ ion in a sweep translates as a jump in the nanotube's differential conductance ${ }^{10,12}$. The corresponding magnetic field, the so-called switching field $\mu_{0} H_{\text {SW }}$ of the $\mathrm{TbPc}_{2} \mathrm{SMM}$, is then extracted from each sweep. Upon repeating the measurement one can construct magnetization reversal histograms as shown in Figs 3 and 4.

The magnetization reversal measurements are carried out in $\mathrm{He}_{3} / \mathrm{He}_{4}$ dilution refrigerator with a base temperature of $20 \mathrm{mK}$. The refrigerator is equipped with two orthogonal magnetic fields coils, generating up to $1.4 \mathrm{~T}$ and up to $0.5 \mathrm{~T}$ in the plane of the sample, with a maximum sweep rate of $250 \mathrm{mT} \mathrm{s}^{-1}$. One can orient the field direction with respect to the SMM's easy axis by probing the magnetization reversal at different magnetic field angles ${ }^{12}$

\section{References}

1. Einstein, A. \& de Haas, W. J. Experimenteller nach-weis der ampereschen molekularstroeme. Deut. Phys. Gesellsch. Verhandlungen 17, 152-170 (1915).

2. Einstein, A. \& de Haas, W. J. Experimental proof of the existence of ampere's molecular currents. KNAW Proc. 18 I, 696-711 (1915).

3. Richardson, O. W. A mechanical effect accompanying magnetization. Phys. Rev. (Series I) 26, 248-253 (1908).

4. Noether, E. Invariante variationsprobleme. Nachr. D. Koenig. Gesellsch. D. Wiss. Zu Goettingen, Math-phys. Klasse 1918, 235-257 (1918).

5. Chudnovsky, E. M. Conservation of angular momentum in the problem of tunneling of the magnetic moment. Phys. Rev. Lett. 72, 3433-3436 (1994).

6. Chudnovsky, E. M. \& Garanin, D. A. Rotational states of a nanomagnet. Phys. Rev. B 81, 214423 (2010).

7. Chudnovsky, E. M., Garanin, D. A. \& Schilling, R. Universal mechanism of spin relaxation in solids. Phys. Rev. B 72, 094426 (2005).
8. Bogani, L. \& Wernsdorfer, W. Molecular spintronics using single-molecule magnets. Nat. Mater. 7, 179-186 (2008).

9. Gatteschi, D. et al. Molecular Nanomagnets (Oxford University Press, 2006).

10. Urdampilleta, M., Klyatskaya, S., Cleuziou, J.-P., Ruben, M. \& Wernsdorfer, W. Supramolecular spin valves. Nat. Mater. 10, 502-506 (2011).

11. Ishikawa, N., Sugita, M. \& Wernsdorfer, W. Quantum tunneling of magnetization in lanthanide single-molecule magnets: bis(phthalocyaninato) terbium and bis(phthalocyaninato)dysprosium anions. Ang. Chem. Int. Ed. 44, 2931-2935 (2005)

12. Ganzhorn, M., Klyatskaya, S., Ruben, M. \& Werns dorfer, W. Strong spinphonon coupling between a single molecule magnet and a carbon nanotube nanoelectrome-chanical system. Nat. Nanotechnol. 8, 165-169 (2013).

13. Urdampilleta, M., Klyatskaya, S., Ruben, M. \& Wernsdorfer, W. Landau-Zener tunneling of a single $\mathrm{Tb}^{3+}$ magnetic moment allowing the electronic read-out of a nuclear spin. Phys. Rev. B 87, 195412 (2013).

14. Chudnovsky, E. M. in Molecular Magnets, NanoScience and Technology. (eds Bartolome, J., et al.) 61-75 (Springer, 2014).

15. Poot, M. \& van der Zant, H. S. J. Mechanical systems in the quantum regime. Phys. Rep. 511, 273-335 (2012).

16. Kovalev, A. A., Hayden, L. X., Bauer, G. E. W. \& Tserkovnyak, Y. Macrospin tunneling and magnetopolaritons with nanomechanical interference. Phys. Rev. Lett. 106, 147203 (2011).

17. Garanin, D. A. \& Chudnovsky, E. M. Quantum entanglement of a tunneling spin with mechanical modes of a torsional resonator. Phys. Rev. X 1, 011005 (2011).

18. Kyatskaya, S. et al. Anchoring of rare-earth-based single-molecule magnets on single-walled carbon nanotubes. J. Am. Chem. Soc. 131, 15143 (2009).

19. Lopes, M. et al. Surface-enhanced raman signal for terbium single-molecule magnets grafted on graphene. ACS Nano 4, 7531-7537 (2010).

20. Landau, L. Zur theorie der energieubertragung. II. Phys. Z. Sowjetunion 2, 46-51 (1932)

21. Zener, C. Non-adiabatic crossing of energy levels. Proc. R. Soc. London A 137, 696-702 (1932).

22. Stueckelberg, E. Theorie der unelastischen stoesse zwischen atomen. Helvet. Phys. Acta 5, 369-422 (1932).

23. Majorana, E. Atomi orientati in campo magnetico variabile. Il Nuovo Cimento 9, 43-50 (1932).

24. Wernsdorfer, W., Bhaduri, S., Vinslava, A. \& Chris-tou, G. Landau-zener tunneling in the presence of weak intermolecular interactions in a crystal of $\mathrm{mn}_{4}$ single-molecule magnets. Phys. Rev. B 72, 214429 (2005).

25. Garanin, D. A. in Molecular Magnets, NanoScience and Technology (eds Bartolome, J. et al.) pp 129-160 (Springer, 2014).

26. Sapmaz, S., Jarillo-Herrero, P., Blanter, Y. a. M., Dekker, C. \& van der Zant, H. S. J. Tunneling in suspended carbon nanotubes assisted by longitudinal phonons. Phys Rev. Lett. 96, 026801 (2006).

\section{Acknowledgements}

This work was partially supported by the EU by the FP7 FET-Proactive project MoQuaS No. 610449 and the Agence Nationale de la Recherche project MolQuSpin, No. ANR-13-BS10. M.G. acknowledges the financial support from the RTRA Nanosciences Foundation. Samples were fabricated in the NANOFAB facility of the Neel Institute. We thank F. Balestro, E. Bonet, T. Crozes, J. P. Cleuziou, E. Eyraud, T. Fournier, R. Haettel, C. Hoarau, D. Lepoittevin, V. Nguyen, V. Reita, S. Thiele, C. Thirion, M. Urdampilleta and R. Vincent.

\section{Author contributions}

M.G. and W.W. designed, conducted and analysed the experiments. S.K. and M.R designed, synthesized and characterized the molecule. M.G. and W.W. co-wrote the paper.

\section{Additional information}

Competing financial interests: The authors declare no competing financial interests.

Reprints and permission information is available online at http://npg.nature.com/ reprintsandpermissions/

How to cite this article: Ganzhorn, M. et al. Quantum Einstein-de Haas effect. Nat. Commun. 7:11443 doi: 10.1038/ncomms11443 (2016).

\section{(i)}

This work is licensed under a Creative Commons Attribution 4.0 International License. The images or other third party material in this article are included in the article's Creative Commons license, unless indicated otherwise in the credit line; if the material is not included under the Creative Commons license, users will need to obtain permission from the license holder to reproduce the material To view a copy of this license, visit http://creativecommons.org/licenses/by/4.0/ 